\title{
Genetic mapping of principal components of canine pelvic morphology
}

\author{
Mark J. Fealey ${ }^{1}$, Joy Li ${ }^{2}$, Rebel J. E. Todhunter ${ }^{1}$, Ursula Krotscheck', Kei Hayashi', Marina J. McConkey ${ }^{1}$, \\ Adam R. Boyko 2,3, Jessica J. Hayward ${ }^{2,3}$ and Rory J. Todhunter ${ }^{1,3^{*}}$
}

\begin{abstract}
Background: Concentrated breeding effort to produce various body structures and behaviors of dogs to suit human demand has inadvertently produced unwanted traits and diseases that accompany the morphological and behavioral phenotypes. We explored the relationship between pelvic conformation and canine hip dysplasia (HD) because purebred dogs which are predisposed, or not, to HD share common morphologic features, respectively. Thirteen unique bilateral anatomical features of the pelvis were measured on 392 dogs of 51 breeds and 95 mixed breed dogs. Principal components (PCs) were derived to describe pelvic morphology. Dogs were genotyped at $\sim 183,000$ single nucleotide polymorphisms and their hip conformation was measured by the Norberg angle and angle of inclination between the femoral neck and diaphysis.
\end{abstract}

Results: No associations reached genome wide significance for the Norberg angle when averaged over both hips. PC1 was negatively correlated with the Norberg angle $(r=-0.31 ; P<0.05)$ but not the angle of inclination $(r=-0.08$; $P>0.05)$. PC1, 2, 4, and 5 differed significantly between male and female dogs confirming pelvic sexual dimorphism. With sex as a covariate, the eigenvector contribution to PC1 reflected the overall size of the pelvis and was significantly associated with the IGF-1 locus, a known contributor to canine body size. PC3, which represented a tradeoff between ilial length and ischial length in which a longer ischium is associated with a shorter ilium, was significantly associated with a marker on canine chromosome 16:5181388 bp. The closest candidate gene is TPK1, a thiamine-dependent enzyme and part of the PKA complex. Associations with the remaining PCs did not reach genome wide significance.

Conclusion: IGF-1 was associated with the overall size of the pelvis and sex is related to pelvic size. Ilial/ischial proportion is genetically controlled and the closest candidate gene is thiamine-dependent and affects birth weight and development of the nervous system. Dogs with larger pelves tend to have smaller NAs consistent with increased tendency toward HD in large breed dogs. Based on the current study, pelvic shape alone was not strongly associated with canine hip dysplasia.

Keywords: Dog, GWAS, Principal component analysis, IGF-1, Pelvic sexual dimorphism, Norberg angle, Hip dysplasia

\section{Plain English Summary}

Concentrated breeding effort to produce various body structures and behaviors to suit human demand has inadvertently produced unwanted traits and diseases that accompany the external appearance and behavior of dogs. Purebred dogs, which are predisposed, or not, to

\footnotetext{
*Correspondence: rjt2@cornell.edu

'Department of Clinical Sciences, College of Veterinary Medicine, Cornell University, Ithaca, NY 14853, USA

${ }^{3}$ Cornell Veterinary Biobank, College of Veterinary Medicine, Cornell

University, Ithaca, NY 14853, USA

Full list of author information is available at the end of the article
}

HD share common features of their shape and size, respectively. Thirteen unique anatomical features of the pelvis were measured on radiographs of 392 dogs of 51 breeds and 95 mixed breed dogs. Combinations of these measurements together described the shape and size of the pelvis. Male dogs had significantly larger pelves than female dogs. Genetic markers pointed to insulin-like growth factor-1 as a major driver of pelvic size. A second genetic marker was associated with ilial length and ischial width on canine chromosome 16. Conclusion: Based on the current study, pelvic shape alone was not strongly associated with canine hip dysplasia. 


\section{Background}

The domestic dog is arguably the most morphologically diverse mammal [1]. The vast differences in morphology within the species have suggested that genetic variation can rapidly change anatomical features, some of which are related to deleterious traits. Breeds as diverse as Chihuahuas, Great Danes, Salukis, and Bulldogs are all descended from the gray wolf, and are the product of selection that began when the dog derived from the wolf about 15,000 years ago but exact timelines remain elusive [2]. The formation of modern dog breeds began about 200 years ago [3]. The speed and coherence with which these functional adaptations have occurred suggests that selection may be acting on genetic loci that control multiple morphological structures.

The selection of certain morphologic features is likely correlated with the selection of genes that predispose dogs to orthopedic diseases. Breeds such as the American Bulldog and Saint Bernard, which are large and stocky, have an increased propensity to develop hip dysplasia (HD) than breeds such as the Greyhound, Saluki or Borzoi [4]. Hip dysplasia is the abnormal development of the coxofemoral joint(s). Joint laxity is generally considered to be one of the earliest pathologic findings in $\mathrm{HD}$ and is a major precursor for the osteoarthritic changes that are typically associated with HD $[5,6]$. The precise genetic factors that initiate HD are unknown and the rate and extent of its development are variable. Since the first report of HD in the dog in 1935, the disorder has become one of the most commonly diagnosed canine orthopedic diseases [7].

The polygenic mode of inheritance of HD has made reduction in its prevalence slow [8]. The inability to identify the specific genes responsible for the predisposition to HD has, until recently, left only phenotypic evaluation by radiography for the screening of individuals. The expression of polygenic traits is modified by environmental influences thus reducing the proportion of phenotypic variance that has a genetic basis [8]. Heritability of HD ranges from 0.2 to 0.6 [9-13]. Because morphology and orthopedic disease are intertwined, probing the genetic basis of pelvic morphology may shed light on the genetic basis of the HD here measured by the Norberg angle.

Chase et al., [14] described the relationships of what they referred to as "tradeoffs" in pelvic morphology of the Pit Bull Terrier type dog and the Greyhound. When the ratio of the length of the long bones to the width of the cranium and the ratio of the muscle mass of the hind limbs to the diameter of the femur increases, the susceptibility to HD decreases. Morphotypes like the Pit Bull Terrier and American Bulldog are predisposed to HD while Greyhounds, Borzois and Salukis are less susceptible to HD (http\offa.org) [9, 15]. Because dogs with HD have disease in other joints [16-19] and the acetabulum is part of the pelvis, it behooves the question: are there morphological features of pelvic shape that contribute to HD? Chase et al., [14] point out that quantitative trait loci (QTLs) related to pelvic shape may be relevant to disease. They found that a particular haplotype of the QTL associated with the simple sequence repeat marker FH2388 was associated with osteoarthritis (OA) in the coxofemoral joint that resulted from HD in Portuguese Water Dogs. This marker, they also discovered, was associated with pelvic shape. They proposed that the acetabular OA appeared to result directly from the action of the QTL haplotype rather than indirectly from changes in pelvic shape produced by the QTL [20].

To assess the relationship of the femoral head and proximal femur with the acetabulum and pelvis, we measured the Norberg angle and the angle of inclination of the femoral neck to the femoral diaphysis in dogs. The Norberg angle [21] measures the coverage of the femoral head by the acetabulum with the femora in an extended position and the dog lying in dorsal recumbency. The angle of inclination has been used to assess normal and dysplastic conformation in dogs but its relationship to HD has been equivocal [22-25].

One method to explore the relationship between many measurements on the same organ is through principal component analysis (PCA), which classifies phenotypic variation into independent systems of correlated traits [26]. Individual dogs each have a value for every principal component (PC). Principal components of pelvic shape are heritable [14]. Thus, PCs are phenotypes that can be subjected to genetic analysis, and QTLs can be identified that inform these phenotypes. As Chase et al., [14] elegantly explained, the genetics of PCs can be used to dissect genetic networks that regulate complex biological systems like pelvic shape [14] and in so doing, we may discover variants that contribute to HD or protect against it.

The aims of this study were to use PCA to reduce morphologic features of the canine pelvis into a set of independent variables and then to map these PCs in a genome wide association study. Our hypothesis was that QTLs would be associated with the PCs of these pelvic measurements. Genes in these QTLs may contribute to HD because the coxofemoral joint is an integral part of the pelvic architecture so that pelvic conformation and HD are inextricably linked.

\section{Methods}

Dogs

Pelvic dimensions were measured from digital or hard copy radiographs from the following sources: the Cornell University Hospital for Animals, the Baker Institute for Animal Health at Cornell University, and the Guiding Eyes for the Blind in Yorktown Heights, NY. A total of 530 dogs were measured but all measurements were 
available on 392 dogs, which formed the cohort for this study. The majority of which were German Shepherd (6\% $[n=25])$, Golden Retriever (9\% $[\mathrm{n}=36])$, Labrador Retriever (35\% [ $n=137]$ ), and Greyhound/ Labrador Retriever cross breed dogs $(24 \%[n=95])$ (Table 1$)$.

\section{Radiographic measurements}

Thirteen pelvic dimensions (pubis to ischium, left ischial length, right ischial length, span of cranial ilium, width of sacrum, left ilial length, right ilial length, left ischial tuberosity length, right ischial tuberosity length, left os coxa length, right os coxa length, pelvic inlet diameter, internal pelvic angle) were measured in millimeters on a pelvic radiograph, with the dog in one of four different positions: ventrodorsal extended hip (Fig. 1), ventrodorsal frog-leg, ventrodorsal PennHip $^{\text {Ts }}$, or the dorsolateral subluxation position. Forty randomly selected radiographs were remeasured to assess repeatability of the measurements. The measurements were summarized descriptively using the stat.desc function in the pastecs package in R [27]. The Norberg Angle (NA) was measured for each hip of these dogs, and then averaged. All NA scores below $75^{\circ}$ were set to $75^{\circ}$ to reduce outlier effects. Hip dysplasia was also trichotomized, based on the average Norberg angle, as normal (Norberg angle $>105^{\circ}$ ), indeterminate $\left(95^{\circ}-105^{\circ}\right)$, and dysplastic $\left(<95^{\circ}\right)$ conformation [28]. The angle of inclination was measured on each femur of each dog from the ventrodorsal extended hip radiograph, and then averaged for each dog.

\section{Principal component analysis}

The prcomp function in $\mathrm{R}$ [27] was used to calculate PCs from the 13 measured hip phenotypes for 392 dogs of 51 different breeds and 95 mixed-breed dogs. The 392 dogs had radiographic measurements for all 13 phenotypic traits. The correlation matrix was used in the PCA to account for the different dog sizes, scales, and magnifications of the radiographic measurements.

Body weights (expressed as body weight ${ }^{0.303}$ based on a Box-Cox transformation to normalize the distribution of weights across breeds) were available for 188 of the 392 dogs. We regressed body weight against each PC to

Table 1 Breed summary of Norberg Angle (NA) in degrees expressed as the mean (standard deviation)

\begin{tabular}{lllll}
\hline Breed & $\begin{array}{l}\text { No. } \\
\text { dogs }\end{array}$ & Left & Right & Average \\
& NA & NA \\
\hline German shepherd dog & 25 & $91.5(11.7)$ & $92.3(11.2)$ & $91.9(10.7)$ \\
Golden retriever & 36 & $92.1(10.5)$ & $92.9(12.6)$ & $92.3(11.3)$ \\
Labrador retriever & 137 & $103.8(9.2)$ & $104.4(9.2)$ & $104.1(8.8)$ \\
Newfoundland & 15 & $91.1(11.5)$ & $96.9(9.2)$ & $94.0(9.9)$ \\
Labrador/Greyhound cross & 95 & $106.8(5.8)$ & $107.7(4.9)$ & $107.2(4.7)$ \\
Other & 82 & $96.6(12.2)$ & $98.9(12.3)$ & $97.8(11.9)$ \\
Total & 397 & & & \\
\hline
\end{tabular}

determine significant relationships in this group of dogs. Correlation analysis was also used to determine if the average Norberg angle or the average angle of inclination was related to any of the PCs.

\section{Genome-wide association study}

The dogs used in the pelvic PCA were genotyped as part of a complex trait mapping study [29]. Briefly, genotyping was done using the Illumina 170k CanineHD array, with the addition of 12,143 custom markers (see PLINK genotype files used in that paper by Hayward et al. [29] that are deposited in Dryad) producing an array composed of $\sim 183,000$ markers. The genotyping methods are exactly as described [29]. Using PLINK v1.07 [30], 180,117 single nucleotide polymorphisms (SNPs) remained after filtering (removal of SNPs with a genotyping rate $<95 \%$, that deviated from Hardy-Weinberg equilibrium, or that were discordant between duplicate samples), with an overall call rate of $>99.8 \%$. Phasing was done for all autosomal and X chromosome markers, and additional custom plates or CanineHD datasets were pre-phased using SHAPEIT [31], and then phased with IMPUTE2 [32].

For the genome wide association study (GWAS), the PCs, the Norberg angle averaged over each dog, and the angle of inclination averaged over each dog were analyzed in a linear mixed model framework using the program GEMMA v.0.94 [33]. Only SNPs with minor allele frequency (MAF) $>0.05$ were included in the analysis and a significance threshold of $P<3.5 \times 10^{-7}$ (the Bonferroniadjusted genome wide $P$-value $<0.05$ ) was used. Because pelvic sexual dimorphism has been described in dogs previously [34], we used a $t$-test to determine if there was a difference between the sexes for each $\mathrm{PC}$, and then included sex as a covariate in the GWAS for those PCs that were significantly different $(P<0.05)$ according to sex.

\section{Results \\ Dogs}

The 13 radiographic measurements are summarized in Table 2. The Norberg angle and angle of inclination were analyzed separately for their relevance to hip conformation and HD and were not included in the PCA. The Norberg angle and the internal pelvic angle were significantly correlated $(r=-0.17, \quad P<0.001)$, but the angle of inclination was not significantly correlated with either the Norberg angle or the internal pelvic angle $(r=$ $0.042, P=0.21$ and $r=0.048, P=0.18$, respectively). The correlation coefficient between the five bilateral measurements ranged from 0.6 to 0.96 . The angle of inclination correlation between left and right was 0.6. This is not surprising because each hip of each dog varies by natural asymmetry and HD. For 20 randomly selected repeat digital radiographic measurements, the correlation coefficients ranged from 0.69 to 0.97 . For 20 randomly 


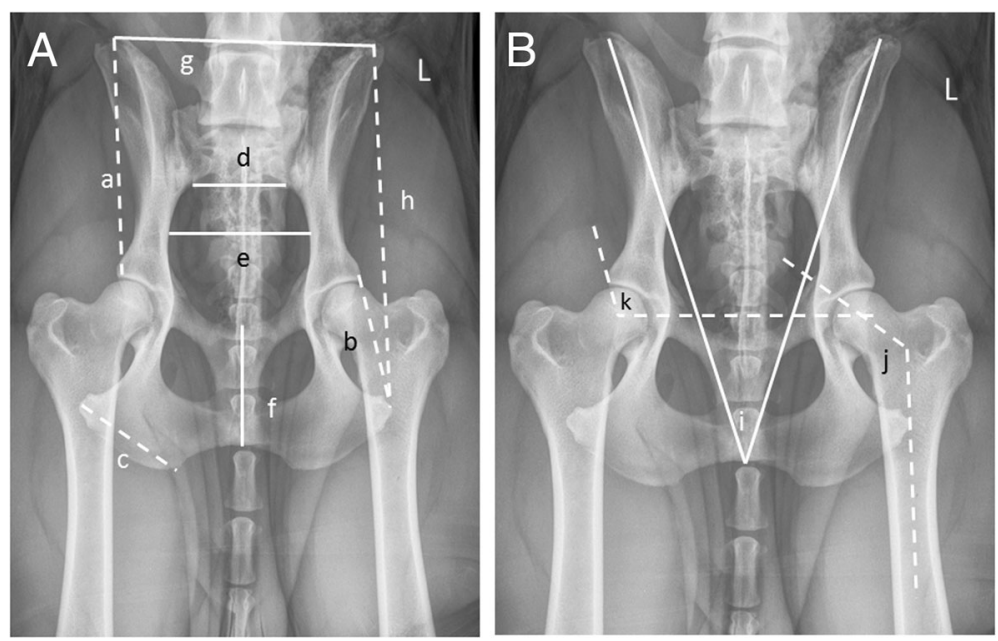

Fig. 1 a Ventrodorsal radiograph of a pelvis with measurement labels for ilial length (a), ischial length $(b)$, ischial tuberosity length (c), width of sacrum (d), pelvic inlet diameter $(e)$, pubis to ischium $(f)$, cranial ilial span $(g)$, and os coxa length $(h)$. b This image shows the internal pelvic angle $(i)$, angle of inclination $(j)$, and Norberg angle $(k)$. For abaxial measurements, both the left and right sides were measured and bilateral measurements are indicated by dashed lines

selected repeat hard copy radiographic measurements, the correlation coefficients ranged from 0.42 to 0.98 . Two dogs had the original right and left os coxa lengths used for the respective ilial lengths reducing the correlation coefficient to 0.42 . Thus two dogs had incorrect individual eigenvalues for PC3. The pelvic measurements of all 530 dogs are provided in Additional file 1: Table S1 and the subset of 392 dogs in Additional file 2: Table S2.

\section{Principal component analysis}

The first eigenvector explained $72 \%$ of the overall variance and the first four vectors explained $90 \%$ of the overall variance (Table 3). The first PC was composed about equally of all the pelvic measurements except the internal pelvic angle (Fig. 1), which was the major contributor to PC2 (Table 4). Body weight was strongly correlated with PC1 $(r=0.79, P<0.0001)$ and weakly correlated with PC2 $(r=0.14, P=0.02)$, PC7 $(r=0.21$, $P=0.002)$ and PC8 $(r=0.18, P=0.007)$. The breeds roughly clustered by body weight (PC1) (Additional file 3: Figure S1A and B). We further showed that the major breeds can be distinguished based on their PC1 values but not based on their PC3 values (Additional file 4: Figure S2). Therefore, body size is a major contributor to $\mathrm{PC} 1$ but not to $\mathrm{PC}$.

Consistent with pelvic morphology displaying sexual dimorphism [34], male $\mathrm{PC1}$ was significantly higher than female PC1 $(\mathrm{t}=4.35, P<0.0001)$. When each $\mathrm{PC}$ was

Table 2 Descriptive statistics summary of the 13 radiographic measurements ( $\mathrm{mm}$ ) shown in Fig. 1

\begin{tabular}{|c|c|c|c|c|c|c|c|}
\hline & $\begin{array}{l}\text { pubis to ischium } \\
\text { (f) }\end{array}$ & $\begin{array}{l}\text { left ischial length } \\
\left(b^{\prime}\right)\end{array}$ & $\begin{array}{l}\text { right ischial length } \\
\text { (b) }\end{array}$ & $\begin{array}{l}\text { span of cranial ilium } \\
\text { (g) }\end{array}$ & $\begin{array}{l}\text { width of sacrum } \\
\text { (d) }\end{array}$ & $\begin{array}{l}\text { left ilial length } \\
\left(a^{\prime}\right)\end{array}$ & $\begin{array}{l}\text { right ilial length } \\
\text { (a) }\end{array}$ \\
\hline Min & 23.1 & 27.5 & 28.7 & 59.0 & 24.0 & 50.4 & 51.9 \\
\hline Max & 67.4 & 106.9 & 105.8 & 152.9 & 65.0 & 152.0 & 152.0 \\
\hline Mean & 46.1 & 57.1 & 57.7 & 110.1 & 45.6 & 101.8 & 101.0 \\
\hline Median & 46.0 & 56.1 & 56.3 & 109.6 & 45.0 & 101.0 & 100.6 \\
\hline \multirow[t]{2}{*}{ Std dev } & 7.1 & 8.7 & 8.9 & 14.8 & 6.4 & 12.3 & 12.4 \\
\hline & $\begin{array}{l}\text { left ischial tuberosity } \\
\text { length }\left(c^{\prime}\right)\end{array}$ & $\begin{array}{l}\text { right ischial tuberosity } \\
\text { length }(c)\end{array}$ & $\begin{array}{l}\text { left os coxa } \\
\text { length (h) }\end{array}$ & $\begin{array}{l}\text { right os coxa } \\
\text { length ( } h \text { ') }\end{array}$ & $\begin{array}{l}\text { pelvic inlet } \\
\text { diameter (e) }\end{array}$ & $\begin{array}{l}\text { internal pelvic } \\
\text { angle (i) }\end{array}$ & \\
\hline Min & 22.1 & 24.2 & 84.8 & 61.0 & 30.7 & 29.0 & \\
\hline Max & 66.9 & 74.4 & 232.7 & 226.7 & 85.7 & 43.0 & \\
\hline Mean & 47.1 & 46.8 & 155.1 & 154.5 & 60.4 & 36.2 & \\
\hline Median & 46.4 & 45.4 & 152.8 & 152.1 & 59.2 & 36.0 & \\
\hline Std dev & 5.7 & 5.7 & 19.9 & 20.3 & 8.0 & 2.4 & \\
\hline
\end{tabular}

The corresponding identifiers for each measurement in Fig. 1 are included next to each measurement. The corresponding bilateral measurement is indicated by the letter prime. All bilateral measurements are indicated as dashed lines in Fig. 1 
Table 3 Eigen values, variances, and cumulative variances for the principal components (PCs) of 13 pelvic measurements summarized in Table 2

\begin{tabular}{lccl}
\hline $\begin{array}{l}\text { Principal } \\
\text { component }\end{array}$ & Eigenvalue & Variance & $\begin{array}{l}\text { Cumulative } \\
\text { variance }\end{array}$ \\
\hline PC1 & 9.39 & 72.19 & 72.19 \\
PC2 & 1.15 & 8.86 & 81.05 \\
PC3 & 0.65 & 4.97 & 86.02 \\
PC4 & 0.52 & 4.00 & 90.02 \\
PC5 & 0.34 & 2.65 & 92.67 \\
PC6 & 0.29 & 2.21 & 94.88 \\
PC7 & 0.19 & 1.47 & 96.35 \\
PC8 & 0.18 & 1.38 & 97.73 \\
PC9 & 0.10 & 0.78 & 98.50 \\
PC10 & 0.09 & 0.72 & 99.22 \\
PC11 & 0.05 & 0.37 & 99.59 \\
PC12 & 0.04 & 0.34 & 99.93 \\
PC13 & 0.01 & 0.07 & 100 \\
\hline
\end{tabular}

plotted against another as a function of HD classified as dysplastic, normal, and indeterminate by the Norberg angle groupings, there was no clustering or differentiation between the three classes according to the PC axes (Fig. 2). Male dogs also had significantly different $\mathrm{PC} 2(\mathrm{t}=2.77, P$ $=0.006)$, PC4 $(\mathrm{t}=2.69, P=0.007)$ and PC5 $(\mathrm{t}=2.12, P=$ $0.034)$ values compared to female dogs. Thus, sex was included as a covariate for the GWAS modeling of PC1, 2, 4, and 5. PC1 $(r=-0.31, P<0.0001)$ and PC7 $(r=0.30, P<$ $0.0001)$ and to a lesser extent PC2 $(R=0.09, P=0.043)$, had modest, but significant, correlations with the NA. Only PC7 was significantly correlated with average angle of inclination $(r=0.23, P<0.0001)$.
The contribution of each measurement to PC3 showed that it represented a tradeoff between ilial length and the length of the ischiatic tuberosity; dogs with shorter ilial lengths have longer/wider ischiatic tuberosities and vice versa. The length of the left and right ischial tuberosities was the major contributor to PC4, sacral width to PC5, length of the pubis to $\mathrm{PC6}$, and pelvic inlet diameter to PC7. Principal components 8 and 9 were reflective of right and left pelvic asymmetry. Principal component 10 was composed predominantly of the span of the cranial ilium.

\section{Genome wide association study}

No associations were found for the Norberg angle averaged over both hip joints or the angle of inclination averaged over both hip joints at a genome wide level of significance. Associations with two PCs reached genome-wide significance (Table 5). With sex as a covariate, GWAS for PC1 had the strongest association at 15:41,229,597 bp, which is the locus for IGF-1 $\left(P=1.9 \times 10^{-8}\right)$ (Fig. 3). IGF-1 is a major growth regulator. Supported by the significant relationship between sex and body weight, PC1 reflects overall size of the pelvis.

The strongest association with PC2 was at 26:15,666,332 $\left(P=5.8 \times 10^{-7}\right)$, which did not reach genome-wide significance. GWAS of PC3 yielded a significant association at 16:5,181,388 $\left(P=1.9 \times 10^{-7}\right)$ (Fig. 4a). This association is located $76 \mathrm{~kb}$ upstream of the candidate gene thiamine pyrophosphokinase 1 (TPK1) (Fig. 4b). Principal Components 4, 5, 6, 7 and 8 were not associated significantly with any loci, although the association with PC7 was just on the Bonferroni adjusted level of genome wide significance.

Even though the QQ plots showed that the likelihood of false positive associations was well controlled as shown in Fig. 3, we also undertook the GWAS after removing the most related dogs. We removed all

Table 4 Composition of the 13 principal components (PCs) with each individual pelvic measurement weighting

\begin{tabular}{lccccccccccccc}
\hline Measurement location & PC1 & PC2 & PC3 & PC4 & PC5 & PC6 & PC7 & PC8 & PC9 & PC10 & PC11 & PC12 & PC13 \\
\hline Pubis to Ischium & 0.28 & 0.02 & 0.32 & 0.04 & -0.04 & 0.66 & 0.44 & -0.36 & 0.01 & -0.22 & -0.04 & 0.055 & 0.01 \\
L.Ischium & 0.21 & 0.14 & 0.44 & 0.18 & 0.11 & -0.33 & 0.10 & 0.21 & -0.13 & -0.04 & 0.33 & 0.58 & 0.18 \\
R.Ischium & 0.29 & 0.12 & 0.44 & 0.21 & 0.12 & -0.29 & 0.03 & 0.15 & -0.09 & -0.09 & -0.50 & -0.50 & -0.16 \\
Span of Cranial Ilium & 0.30 & -0.22 & -0.14 & 0.11 & 0.13 & 0.18 & 0.16 & 0.15 & -0.15 & 0.81 & -0.20 & 0.10 & 0.04 \\
Width of Sacrum & 0.27 & -0.17 & -0.10 & 0.01 & -0.88 & -0.24 & 0.23 & 0.05 & 0.07 & -0.01 & -0.01 & -0.03 & -0.00 \\
L.llium & 0.29 & 0.19 & -0.47 & 0.10 & 0.16 & -0.07 & 0.22 & -0.01 & -0.38 & -0.19 & 0.24 & -0.35 & 0.47 \\
R.llium & 0.28 & 0.21 & -0.51 & 0.09 & 0.18 & -0.11 & 0.15 & -0.01 & 0.13 & -0.25 & -0.30 & 0.38 & -0.47 \\
L.Ischial Tuberosity & 0.28 & 0.045 & -0.01 & -0.45 & -0.03 & 0.37 & -0.20 & 0.71 & 0.05 & -0.19 & 0.02 & -0.03 & 0.01 \\
R.Ischial Tuberosity & 0.25 & 0.03 & 0.09 & -0.80 & 0.13 & -0.31 & 0.06 & -0.39 & -0.00 & 0.14 & -0.05 & 0.01 & 0.03 \\
L.Oscoxa & 0.32 & 0.06 & 0.02 & 0.11 & 0.04 & 0.02 & -0.17 & -0.10 & 0.13 & 0.18 & 0.64 & -0.31 & -0.53 \\
R.Oscoxa & 0.31 & 0.09 & -0.05 & 0.18 & 0.06 & 0.00 & -0.31 & -0.15 & 0.73 & 0.05 & -0.09 & -0.02 & 0.45 \\
Pelvic Inlet Diameter & 0.29 & -0.22 & -0.02 & 0.10 & -0.13 & 0.11 & -0.68 & -0.29 & -0.46 & -0.14 & -0.14 & 0.16 & -0.00 \\
Internal Pelvic Angle & 0.10 & -0.86 & -0.02 & 0.02 & 0.30 & -0.14 & 0.15 & 0.06 & 0.14 & -0.29 & 0.08 & -0.04 & -0.01 \\
\hline
\end{tabular}

Each measurement is shown on radiographs in Fig. 1 


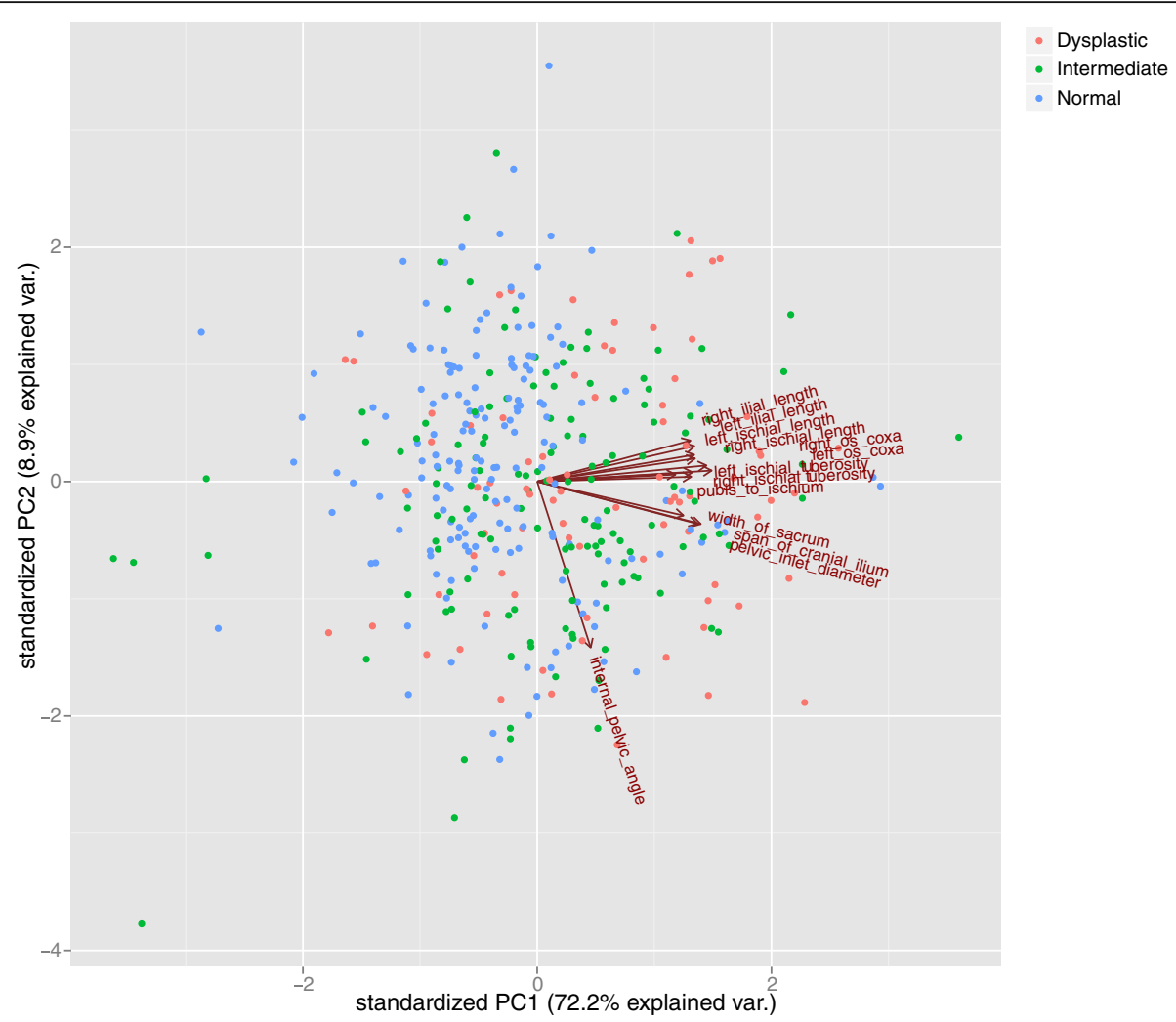

Fig. 2 Plot of PC1 and PC2 values for dysplastic (red) and normal (blue) dogs and for dogs with the intermediate phenotype (green)

Labrador Retriever, Greyhound and Greyhound/Labrador Retriever cross (mix) breed dogs that were related (pi-hat $>0.5$ ), leaving only 25 of these 167 dogs. We then performed the PCA with these related dogs removed and repeated the GWAS (Additional file 5: Table S3). As shown, the PC3 association fell above the threshold for genome-wide significance, but this is not surprising given that this association is driven by Labrador

Table 5 Results from genome wide association study performed on the first eight principal components

\begin{tabular}{llllll}
\hline $\begin{array}{l}\text { Principal } \\
\text { component (PC) }\end{array}$ & Chr & $\begin{array}{l}\text { Marker } \\
\text { position (bp) }\end{array}$ & MAF & Beta & $P$ value \\
\hline PC1 $^{\text {a }}$ & 15 & 41229597 & 0.17 & -1.34 & $1.93 \times 10^{-8 b}$ \\
PC2 $^{\text {a }}$ & 26 & 15666332 & 0.05 & -0.80 & $5.75 \times 10^{-7}$ \\
PC3 & 16 & 5181388 & 0.22 & -0.35 & $1.91 \times 10^{-7 b}$ \\
PC4 & 5 & 36523896 & 0.04 & -0.62 & $2.47 \times 10^{-6}$ \\
PC5 & 1 & 91463706 & 0.27 & 0.22 & $6.67 \times 10^{-7}$ \\
PC6 & 34 & 5001347 & 0.09 & -0.33 & $3.51 \times 10^{-7}$ \\
PC7 & 26 & 8569325 & 0.28 & -0.15 & $1.79 \times 10^{-5}$ \\
PC8 & 31 & 29430539 & 0.07 & -0.32 & $8.88 \times 10^{-7}$ \\
\hline
\end{tabular}

${ }^{a}$ with sex included as a covariate

${ }^{b}$ significant after Bonferroni correction (alpha $=0.05 ; P<3.5 \times 10^{-7}$ )

$\mathrm{Chr}$ is the chromosome number; $\mathrm{bp}$ is base pairs; MAF is minor allele frequency; Beta is the test statistic of the Wald test for the strength of the association
Retrievers and the Greyhound/Labrador Retriever crossbreed (mix) dogs (Table 6). In contrast, the association with the PC1 variant is driven by the breeds other than the major breeds (Table 6).

\section{Discussion}

This sample of purebred and mixed-breed dogs is a subset of dogs that were genotyped previously for complex and fixed trait mapping [29]. Among the dogs in the larger study, 921 had Norberg angle measurements and, of these, we measured pelvic dimensions on 392 dogs. Principal component 1 , when analyzed with sex in the model as a covariate, was associated with a locus that marks IGF-1. Insulin-like growth factor-1 is a major local growth factor that mediates chondrocyte proliferation and hypertrophy [35] as well as osteoblast and osteoclast behavior. Two copies of the derived allele of $I G F-1$ are associated with small stature in dogs $[15,36]$. That male dogs possess larger pelves has been reported based on 20 metrics in Labrador Retrievers [37]. These authors then developed a predictive model based on these measurements that could be applied to discriminate a male from a female canine pelvis in forensic investigations. Most of the pelvis is formed and grows through a process of intramembranous ossification except the acetabulum, which also grows by 


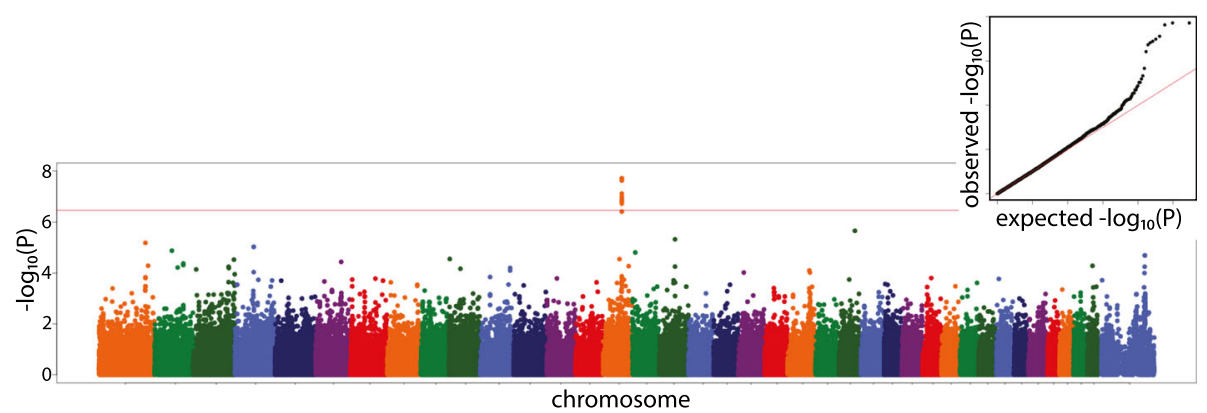

Fig. 3 Manhattan plot of genome wide association study of PC1 modeled with sex as a covariate. Marker position across the chromosome is plotted on the $X$ axis against $\log _{10}(P)$ on the $Y$ axis. The Bonferroni adjusted genome wide threshold is the solid red line drawn across the plot. QQ plot of expected versus observed $-\log _{10}(P)$ is also shown as insert

endochondral ossification. Thus IGF-1 levels influence the size of the pelvic bones through its effects on ossification [38]. The strength of the association with PC1 was not markedly affected by exclusion of the related Greyhound/Labrador Retriever cross breeds because this association was driven by the dog breeds with lower representation (Table 6).

Principal Component 3, representing a trade-off between ilial length and ischial length is consistent with the shorter, broader breeds having wider bodies relative to their body height and length. Breeds with this morphotype, like the Bulldog, Pug, Basset Hound, American Bulldog, French Bulldog, and Pit Bull Terrier are ranked among the worst 30 breeds in frequency of HD in the Orthopedic Foundation for Animals (OFA) HD registry (httploffa.org) and are at higher risk than mixed breed controls [15]. In contrast, the breeds with the lowest frequency of HD in this registry are what Chase and Lark [14] describe as the gracile breeds: Irish Wolfhound, Belgian Tervuren, Irish Setter, Greyhound, Whippet, Italian Greyhound, Saluki, Collie, and Borzoi; breeds with longer ilia relative to the length of their ischiae. Additional file 4: Figure S2 indicates that the major effect of body weight was consumed by the weightings on PC1 and that PC3 was less affected by body weight because the breeds with the most dogs did not separate according to breed (which is related to body size).

The closest candidate gene to the PC3 locus on CFA16 $\left(P=1.9 \times 10^{-7}\right)$ is TPK1, a thiamine-dependent enzyme and part of the protein kinase A $(P K A)$ complex. TPK1 is a cellular enzyme, abundantly expressed in maternal, placental and fetal tissues [39], which catalyzes the conversion of thiamine, a form of vitamin B1, to thiamine pyrophosphate (TPP). TPP is an active cofactor for enzymes involved in glycolysis and energy production, including transketolase, pyruvate dehydrogenase, and alpha-ketoglutarate dehydrogenase [40]. Polymorphisms in this highly conserved enzyme TPK1, were associated with birth weight in neonates and the maternal genotype at the same locus also affected birth weight [40]. No studies are available that compare birth weight to subsequent occurrence of HD. Caloric restriction is known to reduce the incidence and severity of HD [41] and improved glucose handling due to restricted caloric intake improves longevity in dogs [42]. The mechanism of how growth rate influences these processes is unknown. Thiamine is essential for normal development of the nervous system and there is limited research linking muscle and nerve dysfunction in HD [43, 44].

Principal component 2, which was mostly weighted by the internal pelvic angle, had suggestive evidence of association. Dogs with greater acetabular coverage of the femoral head have better hip conformation than those with less coverage and are less prone to secondary OA, the hallmark of prior HD. Because the acetabulum is an integral component of the hemipelvis, we hypothesized that dogs with a more ventroverted hemipelvis would have higher internal pelvic angles and that this would be a feature of breeds resistant to HD. Our hypothesis is supported by analyses of computed tomographs (CT) of babies with developmental dysplasia of the hip (DDH) [45]. Fujii et al.,[35], examined 82 hips of 52 patients and concluded that structural abnormalities exist throughout the pelvis in DDH, and the morphologic abnormalities of the acetabulum are not caused solely by local dysplasia around the hip, but are influenced by the morphologic features of the entire pelvis. They observed greater internal rotation of the innominate bone in patients with DDH than in the control subjects along with increased acetabular anteversion angle and acetabular inclination angle. Internal rotation of the innominate bone also was associated with decreased anterior and superior acetabular coverage [45]. Perhaps the twodimensional nature of a radiograph as suggested by one of the reviewers of our paper, lacked the finesse to capture the finer detail of pelvic rotation and additional measurements made from CT would be more sensitive. 


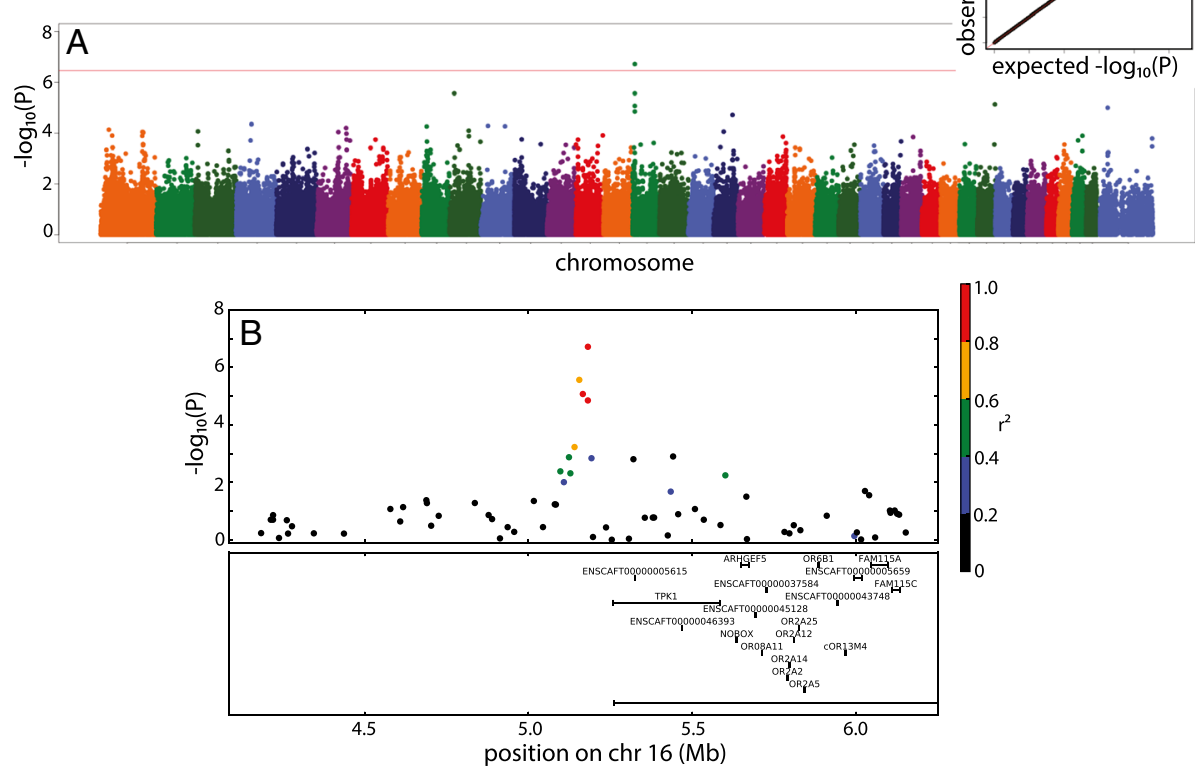

Fig. 4 a Manhattan plot of genome wide association study of PC3. Marker position across the chromosome is plotted on the X axis against $-\log _{10}(P)$ on the $Y$ axis. The Bonferroni adjusted genome wide threshold is the solid red line drawn across the plot. QQ plot of expected versus observed $-\log _{10}(P)$ is also shown as insert. b Manhattan plot of $2 \mathrm{mb}$ region surrounding the associated SNP. Colors of dots indicate the amount of linkage disequilibrium (as measured by $r^{2}$ ) with the associated SNP

Table 6 This table shows details of the breeds contributing to the strength of the association for PC1 on CFA15 (A) and PC3 on CFA16 (B), in the five main breeds and remaining dogs (other)

\begin{tabular}{|c|c|c|c|c|c|c|}
\hline \multicolumn{7}{|l|}{ (A) chr15:41229597 } \\
\hline BREED & $\mathrm{N}$ & Beta & SE & T statistic & $P$ value & $\begin{array}{l}\text { Freq_G } \\
\text { _allele }\end{array}$ \\
\hline Golden Retriever & 36 & 1.223 & 0.833 & 1.468 & 0.151 & 0.056 \\
\hline Labrador Retriever & 137 & -0.363 & 0.309 & -1.173 & 0.243 & 0.347 \\
\hline Mixed Breed & 95 & 0.611 & 0.588 & 1.038 & 0.302 & 0.037 \\
\hline Other & 84 & 2.031 & 0.519 & 3.914 & 0.000186 & 0.344 \\
\hline \multicolumn{7}{|l|}{ (B) $\operatorname{chr} 16: 5181388$} \\
\hline BREED & N & Beta & SE & T statistic & $P$ value & $\begin{array}{l}\text { Freq_A } \\
\text { _allele }\end{array}$ \\
\hline $\begin{array}{l}\text { German Shepherd } \\
\text { Dog }\end{array}$ & 25 & 0.097 & 0.425 & 0.228 & 0.821 & 0.060 \\
\hline Golden Retriever & 36 & -0.219 & 0.130 & -1.684 & 0.101 & 0.444 \\
\hline Labrador Retriever & 137 & -0.434 & 0.133 & -3.277 & 0.0013 & 0.187 \\
\hline Mixed Breed & 95 & -0.325 & 0.166 & -1.951 & 0.054 & 0.132 \\
\hline Newfoundland & 15 & -0.580 & 0.283 & -2.052 & 0.061 & 0.367 \\
\hline Other & 84 & -0.268 & 0.116 & -2.299 & 0.024 & 0.375 \\
\hline
\end{tabular}

Only the major breeds in which all the alleles for each genotype were segregating are shown
We failed to identify polymorphisms associated with pelvic morphology as described by Chase et al., [20]. This may be due to their inclusion of limb morphology, as well as pelvic dimensions, in their PCA or our data set may not have attained sufficient power for associations with the other PCs to reach the genome-wide $P$ value threshold. Chase et al., [20] conducted their morphologic mapping in a single breed, the Portuguese Water Dog. We used a population sample that consisted of many breeds. These breeds and the cross breeds were predominantly medium to large breed dogs. Seventeen QTLs can explain $80-88 \%$ of the variation of body weight and height in individual purebred dogs [29]. The same group also demonstrated that 500-1000 cases and controls are needed to uncover trait or disease loci of moderate effect. Thus, because the pelvic morphology is affected by size and body weight, which themselves are affected by more than 17 loci, it is unlikely that this study contains enough dogs to discover all loci of small to medium effect, especially when background genetics change across all the breeds in this study. Finally, we applied a stringent Bonferroni correction to the experiment-wide $P$ value, some associations were hidden when compared to application of a more liberal false discovery rate. 
However, PCA of 13 measurements of the pelvis across these breeds of dog provided evidence that IGF-1 is associated with the overall size of the pelvis and, as expected, sex is related to pelvic size. We identified an association of the ilial/ischial proportion with a thiamine-dependent, candidate gene. The Norberg angle, a measure of HD in the dog, was moderately correlated with three PCs. Thus, the genes underlying these PCs may also predispose dogs to HD.

\section{Conclusions}

Based on the current study, pelvic shape alone was not strongly associated with caninehip dysplasia.

\section{Additional files}

Additional file 1: Table S1. Excel file of all pelvic measurements of 530 dogs. (XLSX $94 \mathrm{~kb}$ )

Additional file 2: Table S2. Excel file of the subset of 392 pelvic measurements used in this study. (XLSX $57 \mathrm{~kb}$ )

Additional file 3: Figure S1. PCA plot color-coded by breed (A with all breeds included, B with the four main breeds only). The correlation coefficient (r) between PC1 (the PC that is driven by weight) and body weight was 0.79. (PDF $190 \mathrm{~kb}$ )

Additional file 4: Figure S2. Box and whisker plots showing the distribution of $\mathrm{PC}$ values for the two significant associations across the five main breeds. (A) PC1, (B) PC3. (PDF $23 \mathrm{~kb}$ )

Additional file 5: Table S3. Genome wide association study was performed on the first five principal components after removing 142 of the most related (pi-hat > 0.5) Labrador Retriever, Greyhound, and Greyhound/Labrador Retriever (mix) dogs from the analysis. Chr is the chromosome number; bp is base pairs. (DOCX $13 \mathrm{~kb}$ )

\section{Abbreviations}

GWAS: Genome wide association study; HD: Hip dysplasia; IGF-1: Insulin-like growth factor 1; MAF: Minor allele frequency; NA: Norberg angle; PC: Principal component; PCA: Principal component analysis; PKA: Protein kinase A; QTL: Quantitative trait locus/loci; SNP: Single nucleotide polymorphism; TPK1: Thiamine pyrophosphokinase 1

\section{Acknowledgements}

The authors thank the Orthopedic Surgery Service at the Cornell University Hospital for Animals for diagnosis and accompanying DNA samples collected with informed owner consent from the patients. We thank the Cornell Veterinary Biobank team for DNA extraction and sample archiving.

\section{Funding}

The authors thank Zoetis Inc. for a grant to purchase Illumina High Density canine genotyping arrays. This research was supported by NIH grants 5R24 GM082910-03, 3R24GM082910-02S1, and 1R21 AR055228-01A1.

\section{Availability of data and materials}

The genotypes and Norberg angles used in this analysis are a subset of those posted in Dryad (datadryad.org, doi:10.5061/dryad.266k4). The pelvic measurements on all 530 dogs are available in Additional file 1: Table S1 and the pelvic measurements on the final 392 dogs are in Additional file 2: Table S2.

\section{Authors' contributions}

MJF: radiographic measurements, image preparation, and manuscript preparation. JL: data analysis and manuscript preparation. RJET: radiographic measurements, image preparation. UK, KH, MM: case acquisition, manuscript editing, DNA acquisition. ARB: grant writing, genotyping, data analysis. JJH: genotyping, data analysis, manuscript and figure preparation. RJT: concept, manuscript preparation and editing, grant writing, phenotyping, DNA acquisition. All authors read and approved the final manuscript.

\section{Authors' information}

MJF is a fourth year veterinary student in the College of Veterinary Medicine, Cornell University.

$J \mathrm{~L}$ is an undergraduate student assistant in the Department of Biomedical

Sciences, College of Veterinary Medicine, Cornell University.

RJET is an undergraduate student at Cornell University.

UK is an Associate Professor of Surgery in the Department of Clinical

Sciences, College of Veterinary Medicine, Cornell University.

$\mathrm{KH}$ is an Associate Professor of Surgery in the Department of Clinical

Sciences, College of Veterinary Medicine, Cornell University.

MM is a Lecturer in Surgery, in the Department of Clinical Sciences, College of Veterinary Medicine, Cornell University. Her current address is Department of Clinical Sciences, College of Veterinary Medicine, University of Florida. ARB is an Assistant Professor of Genetics in the Department of Biomedical Sciences, College of Veterinary Medicine, Cornell University.

$\mathrm{JJH}$ is a Research Associate in the Department of Biomedical Sciences, College of Veterinary Medicine, Cornell University.

RJT is a Professor of Surgery, in the Department of Clinical Sciences, College of Veterinary Medicine, Cornell University.

\section{Competing interests}

ARB is the chief scientific officer of Embark Veterinary Inc., a partnership with Cornell University, which was established 3 years after this genotyping and phenotyping was performed.

\section{Consent for publication}

Not applicable.

\section{Ethics approval}

Experiments were done with DNA samples and radiographs that were on file and no live animals were directly involved. The original DNA samples were collected with informed owner consent under IACUC approved protocol 2005-0151 for the Cornell Veterinary Biobank.

\section{Publisher's Note}

Springer Nature remains neutral with regard to jurisdictional claims in published maps and institutional affiliations.

\section{Author details}

'Department of Clinical Sciences, College of Veterinary Medicine, Cornell University, Ithaca, NY 14853, USA. ${ }^{2}$ Department of Biomedical Sciences, College of Veterinary Medicine, Cornell University, Ithaca, NY 14853, USA. ${ }^{3}$ Cornell Veterinary Biobank, College of Veterinary Medicine, Cornell University, Ithaca, NY 14853, USA.

Received: 14 December 2016 Accepted: 12 March 2017

Published online: 24 March 2017

\section{References}

1. Evans HE. Miller's anatomy of the dog. 3rd ed. Philadelphia: Saunders; 1993.

2. Vilà C, Savolainen P, Maldonado JE, Amorim IR, Rice JE, Honeycutt RL, Crandall KA, Lundeberg J, Wayne RK. Multiple and ancient origins of the domestic dog. Science. 1997;276:1687-9.

3. Lindblad-Toh K, Wade CM, Mikkelsen TS, Karlsson EK, Jaffe DB, Kamal M, Clamp M, Chang JL, Kulbokas 3rd EJ, Zody MC, et al. Genome sequence, comparative analysis and haplotype structure of the domestic dog. Nature. 2005;438:803-19.

4. Orthopedic Foundation for Animals [ www.offa.org ]. Accessed 1 Jan 2017.

5. Riser WH. The dog as a model for the study of hip dysplasia. Growth, form, and the development of the normal and dysplastic hip joint. Vet Pathol. 1975;12:234-334.

6. Smith GK, Biery DN, Gregor TP. New concepts of coxofemoral joint stability and the development of a clinical stress-radiographic method for quantitating hip joint laxity in the dog. J Am Vet Med Assoc. 1990;196:59-70.

7. Johnson J, Austin C, Breur G. Incidence of canine appendicular musculoskeletal disorders in 16 veterinary teaching hospitals from 1980 through 1989. Vet Comp Orthop Traumatol. 1980;1990(7):56-69. 
8. Leighton EA. Genetics of canine hip dysplasia. J Am Vet Med Assoc. 1997;210:1474-9.

9. Breur GJ, Lambrecht N, Todhunter RJ. The genetics of canine orthopedic traits. In: Hulbert S, Spearing G, Chippendale F, editors. The Genetics of a Dog. Volume 2nd. Oxfordshire: CAB International; 2012. p. 136-53.

10. Sánchez-Molano E, Pong-Wong R, Clements DN, Blott SC, Wiener P, Woolliams JA. Genomic prediction of traits related to canine hip dysplasia. Front Genet. 2015;6:97.

11. Soo M, Sneddon NW, Lopez-Villalobos N, Worth AJ. Genetic evaluation of the total hip score of four populous breeds of dog, as recorded by the New Zealand Veterinary Association Hip Dysplasia Scheme (1991-2011). N Z Vet J. 2015;63:79-85

12. Wilson BJ, Nicholas FW, James JW, Wade CM, Thomson PC. Estimated breeding values for canine hip dysplasia radiographic traits in a cohort of Australian German Shepherd dogs. PLoS One. 2013;8, e77470.

13. Malm S, Sørensen AC, Fikse WF, Strandberg E. Efficient selection against categorically scored hip dysplasia in dogs is possible using best linear unbiased prediction and optimum contribution selection: a simulation study. J Anim Breed Genet. 2013;130:154-64.

14. Chase K, Carrier DR, Adler FR, Jarvik T, Ostrander EA, Lorentzen TD, Lark KG. Genetic basis for systems of skeletal quantitative traits: principal component analysis of the canid skeleton. Proc Natl Acad Sci U S A. 2002:99:9930-5.

15. LaFond E, Breur GJ, Austin CC. Breed susceptibility for developmental orthopedic diseases in dogs. J Am Anim Hosp Assoc. 2002:38:467-77.

16. Burton-Wurster N, Farese JP, Todhunter RJ, Lust G. Site-specific variation in femoral head cartilage composition in dogs at high and low risk for development of osteoarthritis: insights into cartilage degeneration. Osteoarthritis Cartilage. 1999;7:486-97.

17. Farquhar T, Bertram J, Todhunter RJ, Burton-Wurster N, Lust G. Variations in composition of cartilage from the shoulder joints of young adult dogs at risk for developing canine hip dysplasia. J Am Vet Med Assoc. 1997;210:1483-5.

18. Lust G, Williams AJ, Burton-Wurster NI, Beck KA, Rubin G. Effects of intramuscular administration of glycosaminoglycan polysulfates on signs of incipient hip dysplasia in growing pups. Am J Vet Res. 1992;53:1836-43.

19. Olsewski JM, Lust G, Rendano VT, Summers BA. Degenerative joint disease: multiple joint involvement in young and mature dogs. Am J Vet Res. 1983:44:1300-8

20. Chase K, Lawler DF, Carrier DR, Lark KG. Genetic regulation of osteoarthritis: A QTL regulating cranial and caudal acetabular osteophyte formation in the hip joint of the dog (Canis familiaris). Am J Med Genet A. 2005;135:334-5.

21. Comhaire FH, Schoonjans FA. Canine hip dyslasia: the significance of the Norberg angle for healthy breeding. J Small Anim Pract. 2011;52:536-42.

22. Hauptman J, Cardinet GH, Morgan JP, Guffy MM, Wallace LJ. Angles of inclination and anteversion in hip dysplasia in the dog. Am J Vet Res. 1985;46:2033-6.

23. Sarierler M. Comparison of femoral inclination angle measurements in dysplastic and nondysplastic dogs of different breeds. Acta Vet Hung. 2004:52:245-52.

24. Martins J, Ferreira AJ, Ginja MM. Morphometric assessment of the hip joint in the Estrela Mountain Dog breed. Vet Comp Orthop Traumatol. 2012;25:202-10.

25. Banfield CM, Bartels JE, Hudson JA, Wright JC, Hathcock JT, Montgomery RD. A retrospective study of canine hip dysplasia in 116 military working dogs. Part I: angle measurements and orthopedic foundation for animals (OFA) grading. J Am Anim Hosp Assoc. 1996;32:413-22.

26. Jolliffe IT, Cadima J. Principal component analysis: a review and recent developments. Philos Trans A Math Phys Eng Sci. 2016;374:20150202.

27. R: A language and environment for statistical computing. [https://www.Rproject.org/]. Accessed 1 Jan 2017.

28. Verhoeven G, Fortrie R, Van Ryssen B, Coopman F. Worldwide screening for canine hip dysplasia: where are we now? Veterinary surgery : VS. 2012;41:10-9.

29. Hayward JJ, Castelhano MG, Oliveira KC, Corey E, Balkman C, Baxter TL, Casal ML, Center SA, Fang M, Garrison SJ, et al. Complex disease and phenotype mapping in the domestic dog. Nat Commun. 2016;7:10460

30. Purcell S, Neale B, Todd-Brown K, Thomas L, Ferreira MA, Bender D, Maller J, Sklar P, de Bakker PI, Daly MJ, Sham PC. PLINK: a tool set for whole-genome association and population-based linkage analyses. Am J Hum Genet. 2007:81:559-75.

31. Delaneau O, Zagury JF, Marchini J. Improved whole-chromosome phasing for disease and population genetic studies. Nat Methods. 2013;10:5-6.
32. Howie BN, Donnelly P, Marchini J. A flexible and accurate genotype imputation method for the next generation of genome-wide association studies. PLoS Genet. 2009;5, e1000529.

33. Zhao SM, Li WZ, Pan HB, Huang Y, Yang MH, Wei HJ, Gao SZ. Expression levels of candidate genes for intramuscular fat deposition in two Banna mini-pig inbred lines divergently selected for fatness traits. Genet Mol Biol. 2012;35:783-9.

34. Lark KG, Chase K, Sutter NB. Genetic architecture of the dog: sexual size dimorphism and functional morphology. Trends in genetics : TIG. 2006;22:537-44.

35. Wang Y, Menendez A, Fong C, ElAlieh HZ, Chang W, Bikle DD. Ephrin B2/ EphB4 mediates the actions of IGF-I signaling in regulating endochondral bone formation. J Bone Miner Res. 2014;29:1900-13.

36. Sutter NB, Bustamante CD, Chase K, Gray MM, Zhao K, Zhu L, Padhukasahasram B, Karlins E, Davis S, Jones PG, et al. A single IGF1 allele is a major determinant of small size in dogs. Science. 2007;316:112-5.

37. Nganvongpanit K, Pitakarnnop T, Buddhachat K, Phatsara M. Gender-related differences in pelvic morphometrics of the retriever dog breed. Anat Histol Embryol. 2016;46:51-7.

38. Marongiu M, Marcia L, Pelosi E, Lovicu M, Deiana M, Zhang Y, Puddu A, Loi A, Uda M, Forabosco A, et al. FOXL2 modulates cartilage, skeletal development and IGF1-dependent growth in mice. BMC Dev Biol. 2015;15:27.

39. Bellyei S, Szigeti A, Boronkai A, Szabo Z, Bene J, Janaky T, Barna L, Sipos K, Minik $\mathrm{O}$, Kravjak $\mathrm{A}$, et al. Cloning, sequencing, structural and molecular biological characterization of placental protein 20 (PP20)/human thiamin pyrophosphokinase (hTPK). Placenta. 2005;26:34-46.

40. Fradin $D$, Bougneres $P$. Three common intronic variants in the maternal and fetal thiamine pyrophosphokinase gene (TPK1) are associated with birth weight. Ann Hum Genet. 2007;71:578-85.

41. Kealy RD, Lawler DF, Monti KL, Biery D, Helms RW, Lust G, Olsson SE, Smith GK. Effects of dietary electrolyte balance on subluxation of the femoral head in growing dogs. Am J Vet Res. 1993;54:555-62.

42. Larson BT, Lawler DF, Spitznagel ELJ, Kealy RD. Improved glucose tolerance with lifetime diet restriction favorably affects disease and survival in dogs. J Nutr. 2003;133:2887-92.

43. Cardinet GH, Wallace LJ, Fedde MR, Guffy MM, Bardens JW. Developmental myopathy in the canine with type II muscle fiber hypotrophy. Arch Neurol. 1969;21:620-30.

44. Ihemelandu EC, Cardinet 3rd GH, Guffy MM, Wallace LJ. Canine hip dysplasia: differences in pectineal muscles of healthy and dysplastic German Shepherd dogs when two months old. Am J Vet Res. 1983;44:411-6.

45. Fujii M, Nakashima Y, Sato T, Akiyama M, Iwamoto Y. Pelvic deformity influences acetabular version and coverage in hip dysplasia. Clin Orthop Relat Res. 2011;469:1735-42.

\section{Submit your next manuscript to BioMed Central and we will help you at every step:}

- We accept pre-submission inquiries

- Our selector tool helps you to find the most relevant journal

- We provide round the clock customer support

- Convenient online submission

- Thorough peer review

- Inclusion in PubMed and all major indexing services

- Maximum visibility for your research

Submit your manuscript at www.biomedcentral.com/submit
Biomed Central 